\title{
Selective laser trabeculoplasty vs micropulse laser trabeculoplasty in open-angle glaucoma
}

This article was published in the following Dove Press journal: Clinical Ophthalmology

\section{Benjamin Abramowitz' \\ Nisha Chadha ${ }^{2}$ \\ Amr Kouchouk ${ }^{3}$ \\ Rashed Alhabshan ${ }^{4}$ \\ David A Belyea ${ }^{4}$ \\ Tania Lamba ${ }^{4}$}

'Glaucoma Department, Eye Consultants of Northern Virginia, Springfield, VA, USA; ${ }^{2}$ Icahn School of Medicine at Mount Sinai/New York

Eye and Ear, Eye and Vision Research Institute, New York, NY, USA; ${ }^{3}$ Cornea Department, Atlantis Eyecare, Torrance, CA, USA; ${ }^{4}$ Department of Ophthalmology, The George Washington University, Washington, DC, USA
Correspondence: David A Belyea Department of Ophthalmology, The George Washington University, 2150 Pennsylvania Avenue, NW Suite \#2A, Washington, DC 20037, USA Email: dbelyea@mfa.gwu.edu
Aim: The aim of this study was to prospectively compare the efficacy, safety, and tolerability of selective laser trabeculoplasty (SLT) vs micropulse laser trabeculoplasty (MLT) in reducing intraocular pressure (IOP) in open-angle glaucoma patients.

Patients and methods: In all, 38 patients were randomized to $360^{\circ}$ MLT and 31 patients were randomized to $360^{\circ} \mathrm{SLT}$. IOP was measured at intervals of 1 hour and 1, 1-6, 6-12, 6-24, 24-36, and 36-52 weeks. Patients completed a survey 1 week after the procedure. Patients with end-stage, neovascular, uveitic, or angle-closure glaucoma were excluded. Treatment response was defined as an IOP reduction of $\geq 20.0 \%$ or $\geq 3 \mathrm{mmHg}$ from baseline.

Results: IOP was lowered to $\geq 3 \mathrm{mmHg}$ from baseline among $37.0 \%$ of the micropulse patients and $36.0 \%$ of patients in the selective laser group at 24-52 weeks. Similarly, $29.6 \%$ of the micropulse patients and $36.0 \%$ of the selective laser patients experienced a $20.0 \%$ IOP decrease from baseline during the $24-52$-week interval $(P=0.77)$. Both groups revealed similar reductions in IOP as absolute values and percentage decreases from baseline at all intervals up to 52 weeks post treatment. There were more treatment failures in the micropulse group up to 52 weeks post laser treatment; however, this was not statistically significant. The micropulse group reported less pain both during and after the procedure $(P=0.005)$.

Conclusion: Micropulse trabeculoplasty has demonstrated similar efficacy to SLT over a 52-week follow-up period with less discomfort experienced both during and after the procedure.

Keywords: randomized controlled trial, selective laser trabeculoplasty, micropulse, glaucoma, intraocular pressure

\section{Introduction}

Laser trabeculoplasty was first introduced by Wise and Witter in 1979 in the form of argon laser trabeculoplasty (ALT). ${ }^{1}$ The glaucoma laser trial showed that ALT has similar, if not superior, efficacy to topical beta blocker therapy. ${ }^{2}$ In 1995, Latina et al introduced selective laser trabeculoplasty (SLT), which offered intraocular pressure (IOP) reduction similar to ALT with more targeted treatment and less tissue destruction. ${ }^{3,4}$ Since then, studies have demonstrated SLT to be as effective as monotherapy with drops. ${ }^{5}$

In 1990, Pankratov ${ }^{6}$ introduced micropulse laser as an experimental modality for retinal photocoagulation. Since then, extensive studies have demonstrated stimulation of the retinal pigmented epithelium without permanent thermal damage. ${ }^{7}$ Researchers recently applied this therapy to the pigmented trabecular meshwork to determine if IOP could be reduced without the thermal damage of argon laser. ${ }^{8}$ A histologic study supported this theory by confirming the lack of trabecular meshwork destruction with micropulse laser. ${ }^{9}$ 
Clinical results of micropulse laser trabeculoplasty (MLT) were first reported by Ingvoldstad et $\mathrm{al}^{10}$ at the Association for Research in Vision and Ophthalmology meeting in 2005. Since then, a few other studies investigated MLT at various laser wavelengths and degrees of treatment with variable results. ${ }^{11-16}$ The purpose of this study was to evaluate the efficacy of $360^{\circ} 577 \mathrm{~nm}$ MLT as compared to $360^{\circ} \mathrm{SLT}$ in a prospective, randomized manner.

\section{Patients and methods}

A total of 69 patients with uncontrolled open-angle glaucoma were recruited at the George Washington University by two glaucoma attending professors. Research protocol approval was obtained from the institutional review board of George Washington University (Washington, DC, USA) under IRB \#041, 310 and registered on ClinicalTrials.gov with identifier NCT01956942. Written informed consent was received from all patients, and the protocol followed the Declaration of Helsinki.

This study included all laser trabeculoplasty candidates with open-angle glaucoma on maximally tolerated medical therapy with the need for additional IOP lowering. Patients with end-stage, neovascular, uveitic, or angle closure glaucoma were excluded. In all, 38 patients were randomized to MLT and 31 patients to SLT via a random number generator protocol approved for up to 100 patients. All patients had only one eye included in the study. Patients were allocated to each group without any stratification based on prior characteristics via sealed numbers in a set sequence. Recruitment was concluded after a power analysis revealed an $80 \%$ power at a 0.05 $\alpha$ level detecting an average $2.99 \mathrm{mmHg}$ or $16.19 \%$ decrease from baseline between the SLT and MLT groups. This level was determined based on prior studies defining a $\geq 3 \mathrm{mmHg}$ or $\geq 20.0 \%$ decrease in IOP as a significant difference from baseline. ${ }^{11}$ Patients, physicians, and researchers were not blinded to the different laser treatments. Each of the two physicians performed both the laser treatments and all subsequent follow-ups for a particular patient.

The MLT group underwent $360^{\circ}$ treatment with the IQ $577 \mathrm{~nm}^{\mathrm{TM}}$ yellow laser (Iridex Corporation, Mountain View, CA, USA) with laser applied to the pigmented trabecular meshwork in a confluent manner. The laser settings for MLT were 1,000 mW, $300 \mathrm{~ms}$ duration, $300 \mu \mathrm{m}$ spot size, and a $15 \%$ duty cycle. The SLT group received $360^{\circ}$ treatment with the $532 \mathrm{~nm}$ Q-switched, frequency-doubled Nd:YAG Selecta II TM (Lumenis Inc, San Jose, CA, USA). The energy for SLT was titrated, between $\sim 0.6$ and $\sim 2.0 \mathrm{~mJ}$, based on trabecular meshwork pigmentation to achieve a noticeable gas bubble response, utilizing a $400 \mu \mathrm{m}$ spot size and a $3 \mathrm{~ns}$ duration.

All patients were treated with one drop of apraclonidine prior to the treatment. Patients were discharged with topical diclofenac $0.1 \%$ to use as needed for discomfort. At week 1 , patients completed a standardized survey querying discomfort experienced both during and after the procedure and use of the topical diclofenac.

Patients' IOP was measured at predefined intervals of 1 hour and 1-, 6-, 6-12-, 12-24-, 24-36-, and 36-52-week intervals based on a fixed follow-up of 1 and 6 weeks and a tendency to follow these patients every 3-4 months thereafter. Pretreatment IOP values were calculated as the average of up to three preceding visits to reduce fluctuations. Only the preceding visits without the addition of a drop or a laser were included to avoid confounding influences. If patients were examined multiple times within an interval, IOP values were averaged for statistical analysis. Patients' responses were excluded from the analysis if they underwent any interventions that may affect their IOP, including the addition of a topical antihypertensive drop, cataract surgery, cyclophotocoagulation, incisional glaucoma surgery, or further laser trabeculoplasty. However, their data were still included for the intervals up to the time of these interventions. Comparison between groups was calculated with Student's $t$-test for continuous data and a two-tailed Fisher's exact test for categorical data.

\section{Results}

There was no statistically significant difference in demographic characteristics between groups, as seen in Table 1. The MLT patients had a higher average starting IOP (18.26 mmHg) than the SLT group (16.85 $\mathrm{mmHg}$ ), approaching significance $(P=0.08)$. Both groups had very similar 1 hour posttreatment IOP, $15.15 \mathrm{mmHg}$ in the MLT group and $15.65 \mathrm{mmHg}$ in the SLT group. An IOP spike was defined as an elevation of $>5 \mathrm{mmHg}$ from the pretreatment mean. Incidence of post-procedure spikes were $5.3 \%$ and $12.9 \%$ in the MLT and SLT groups, respectively, but this difference was not statistically significant $(P=0.4)$. No group experienced any anterior chamber inflammation upon examination at the 1 week follow-up time point.

Follow-up data at the 1-, 1-6-, 6-12-, 12-24-, 24-36-, and 36-52-week intervals were tabulated as absolute IOP decreases from pretreatment levels (Figure 1) and percentage IOP decreases from baseline (Figure 2). These data demonstrated that both MLT and SLT provided comparable decreases in IOP from baseline as both an absolute measurement and a percentage decrease at all intervals up 
Table I Demographic and prior ocular history in the MLT and SLT groups

\begin{tabular}{llll}
\hline Characteristics & MLT $(\mathbf{n}=\mathbf{3 8})$ & $\mathbf{S L T}(\mathbf{n}=\mathbf{3} \mathbf{I})$ & $\mathbf{P}$-value \\
\hline Gender & $42.0 \%$ females, $58.0 \%$ males & $61.0 \%$ females, $39.0 \%$ males & 0.15 \\
Mean age & 66.1 years & 67.6 years & $0.6 \mathrm{I}$ \\
Lens status & $80.0 \%$ phakic, $2.0 \%$ phakic, & $74.0 \%$ phakic, & 0.78 \\
& $18.0 \%$ pseudophakic & $26.0 \%$ pseudophakic & 0.17 \\
Prior SLT & $21.1 \%$ & $6.0 \%$ & 0.20 \\
Prior ALT & $10.5 \%$ & $22.6 \%$ & 0.62 \\
Prior glaucoma valve surgery & $7.9 \%$ & $3.2 \%$ & 1.0 \\
Prior filtering surgery & $2.6 \%$ & None & 0.68 \\
Average laser spots per session & 111.15 & 112.42 & \\
\hline
\end{tabular}

Notes: There were similar demographics between the groups. The main differences were more males in the MLT group and more females in the SLT group ( $P=0.15)$, along with more prior SLT in the MLT group $(P=0.17)$.

Abbreviations: MLT, micropulse laser trabeculoplasty; SLT, selective laser trabeculoplasty; ALT, argon laser trabeculoplasty.

to 52 weeks $(P>0.05)$. In addition, the confidence intervals at each time period were $<3 \mathrm{mmHg}$ when viewed as an absolute decrease in IOP and $<20.0 \%$ when viewed as a percentage decrease from baseline IOP, except one outlier interval (week 24-36 in Figure 1). A Kaplan-Meier curve is demonstrated in Figure 3. As expected, the larger proportion of failures in the MLT group produced a lower curve, with more of the MLT failures occurring in the early period. However, the survival curve steps in both groups were similar at later intervals, leading to a log-rank $P$-value of 0.31 .

The data from the standardized pain survey (scale of $0=$ none to $10=$ severe) revealed that the MLT patients experienced a mean 1.34/10 during treatment and $0.89 / 10$ during the 1 week posttreatment period, while the SLT patients noted 2.83/10 during treatment and 2.81/10 during the 1 week posttreatment period. The MLT patients on average experienced less pain both during and after the treatment $(P=0.005)$.

Prior authors defined a successful laser trabeculoplasty as $\geq 3 \mathrm{mmHg}$ IOP decrease or $\geq 20.0 \%$ IOP decrease from baseline. ${ }^{6,8}$ Using these criteria, $29.6 \%$ of the MLT and $36.0 \%$ of the SLT patients experienced $\mathrm{a} \geq 20.0 \%$ IOP decrease from baseline during the 24-52-week interval $(P=0.77)$. Similarly, $37.0 \%$ of the MLT patients and $36.0 \%$ of the SLT patients experienced $\mathrm{a} \geq 3 \mathrm{mmHg}$ IOP reduction from baseline at the 24-52-week interval $(P=1.0)$.

Patients in each group were also assessed to determine the proportion that required further intervention for their glaucoma during the 52-week follow-up period, indicating a failure of the laser therapy. In the MLT group, a total of $23.7 \%$ of patients failed and required further treatment for their glaucoma. A topical antiglaucoma agent was added in $15.8 \%$ of the MLT patients, incisional glaucoma surgery was performed in $2.6 \%$ of the MLT patients, and transscleral cyclophotocoagulation was performed in $5.3 \%$ of the MLT patients. In the SLT group, $12.9 \%$ of the patients failed, with all the failures due to the addition of another topical antiglaucoma medication. Although there were a greater number of failures in the MLT group (23.7\%) compared to the SLT group $(12.9 \%)$, the analysis did not meet significance in each individual category or when considered in sum $(P=0.22)$. No patient in either group experienced any complications, other than a transient rise in IOP.

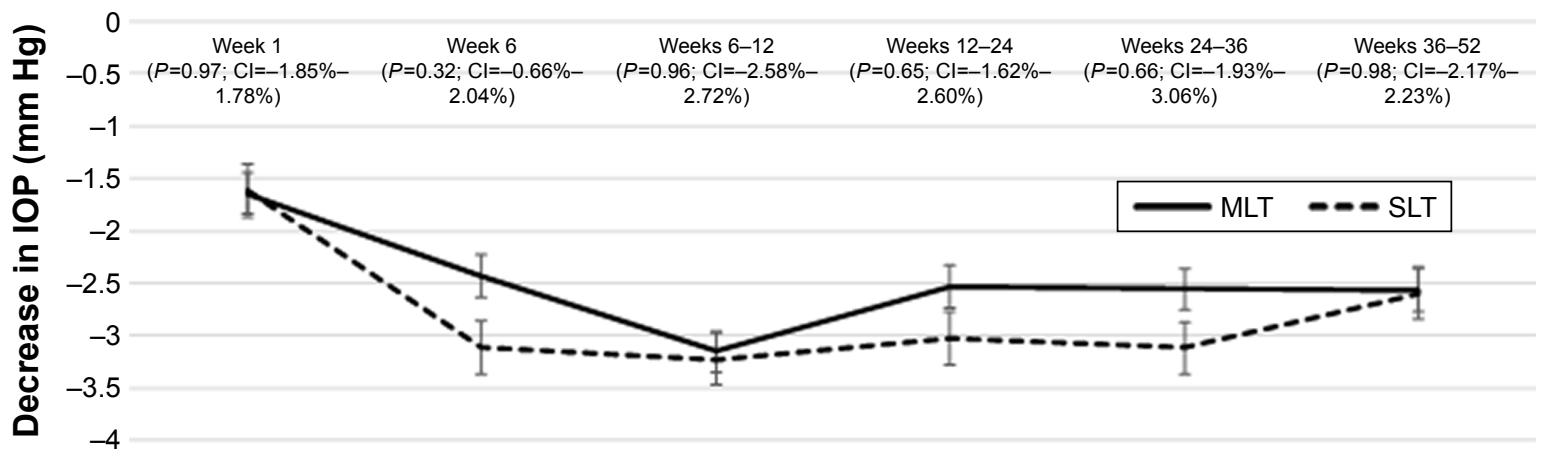

Figure I Absolute decrease in IOP from pretreatment levels in $\mathrm{mmHg}$ between the MLT and SLT groups at follow-up intervals up to I year. Note: $P$-values, $\mathrm{Cl}$, and standard error bars are presented for each interval.

Abbreviations: IOP, intraocular pressure; MLT, micropulse laser trabeculoplasty; SLT, selective laser trabeculoplasty; Cl, confidence interval. 


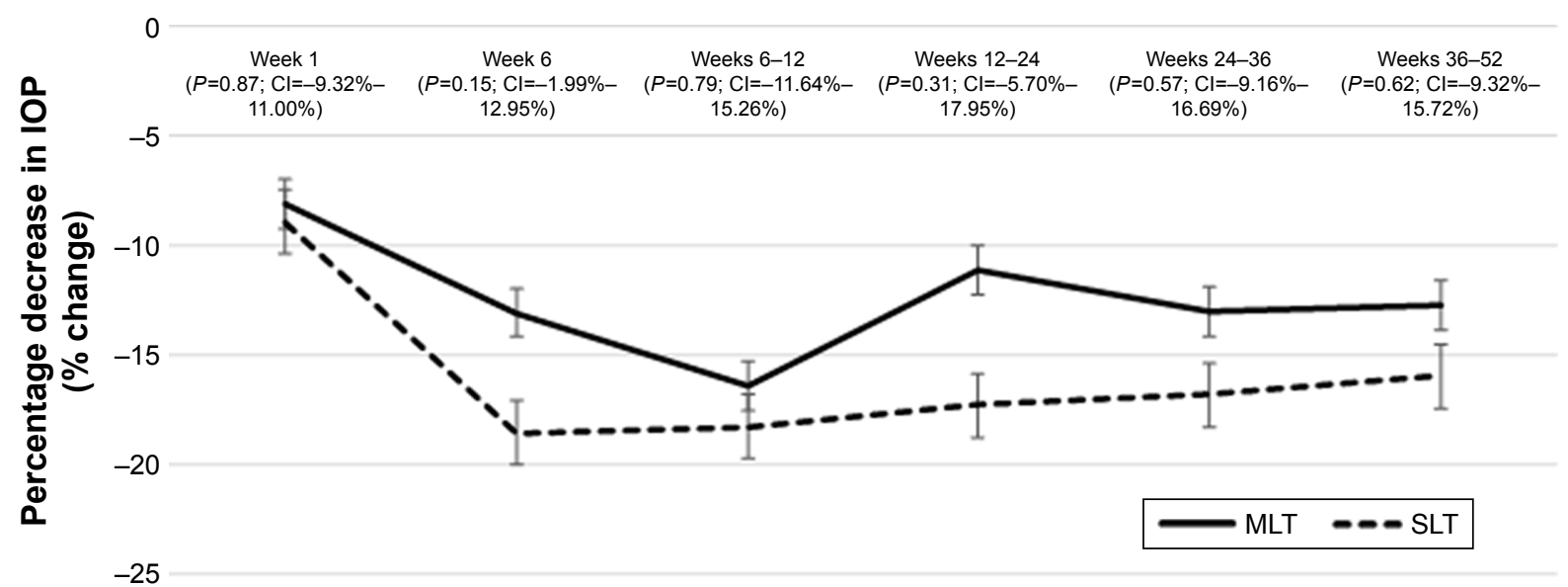

Figure 2 Percentage decrease in IOP from pretreatment levels between the MLT and SLT groups at follow-up intervals up to I year.

Note: $P$-values, $\mathrm{Cl}$, and standard error bars are presented for each interval.

Abbreviations: IOP, intraocular pressure; MLT, micropulse laser trabeculoplasty; SLT, selective laser trabeculoplasty; Cl, confidence interval.

\section{Discussion}

Since Ingvoldstad et al's findings in 2005, several other authors have explored the use of MLT in human patients. ${ }^{10}$ In 2008, Fea et $\mathrm{al}^{11}$ studied a cohort of 20 eyes with MLT applied to $180^{\circ}$ of the trabecular meshwork with a $810 \mathrm{~nm}$ diode laser, noting a mean IOP reduction of $22.1 \%$ after 12 months in the 15 respondent eyes.

In 2012, Rantala and Välimäki ${ }^{12}$ also studied $180^{\circ}$ MLT treatment with the $810 \mathrm{~nm}$ diode laser, concluding that only $2.5 \%$ of patients experienced $\mathrm{a} \geq 20.0 \%$ IOP decrease from baseline and $7.5 \%$ of patients had a $\geq 3 \mathrm{mmHg}$ IOP decrease without further intervention. Conversely, Babalola ${ }^{13}$ utilized the $810 \mathrm{~nm}$ diode laser applied to the inferior $180^{\circ}$ for MLT in a Nigerian population with a sustained IOP lowering effect from weeks to months with an average IOP decrease of $17.2 \%$.

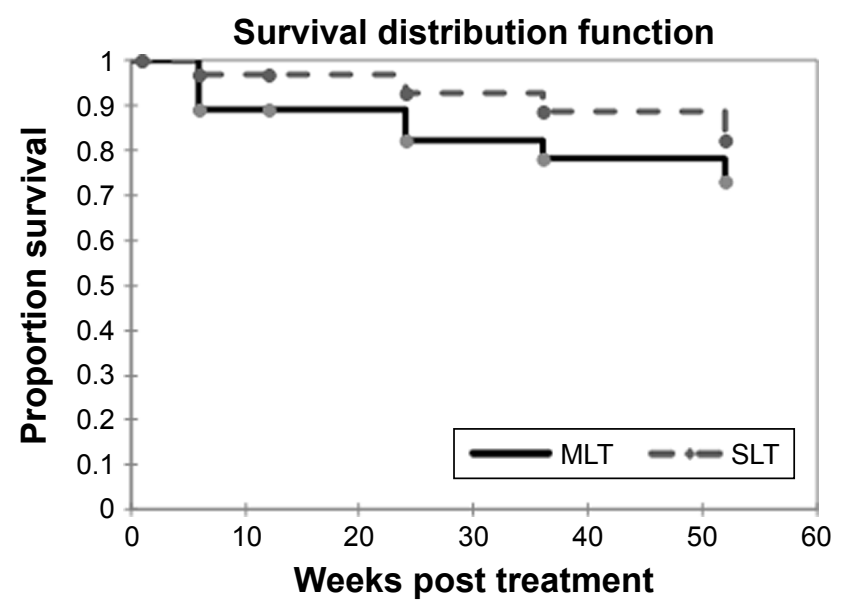

Figure 3 Kaplan-Meier survival curve demonstrating the proportionate failures at each week interval in the MLT and SLT groups.

Abbreviations: MLT, micropulse laser trabeculoplasty; SLT, selective laser trabeculoplasty.
The first prospective, randomized trial comparing MLT to another laser therapy (ALT) was conducted by Detry-Morel et $\mathrm{al}^{14}$ in 2008 utilizing $180^{\circ}$ treatment in each arm with the $810 \mathrm{~nm}$ MLT laser. Both groups experienced a significant decrease from baseline. However, at 3 months of follow-up, the ALT group had a significantly greater reduction in absolute value and percentage decrease from baseline IOP levels compared to the MLT group.

Chudoba et a ${ }^{15}$ initiated a pilot study using a $577 \mathrm{~nm}$ MLT laser to the inferior trabecular meshwork in 2014. This cohort experienced a mean $1.7 \mathrm{mmHg}$ decrease from baseline, while untreated fellow eyes had a decrease in IOP of $1.8 \mathrm{mmHg}$. Recently, Lee et $\mathrm{al}^{16}$ reported in 2015 a $72.9 \%$ success rate for MLT with a $360^{\circ}$ treatment utilizing the $577 \mathrm{~nm}$ laser in achieving $\geq 20.0 \%$ IOP reduction at 1 month post treatment.

The results from our study reveal that both therapies are effective and safe modalities with a similar efficacy as shown in Figures 1 and 2. The success rates for MLT were higher than some previously reported studies involved in achieving the goal of $\geq 3 \mathrm{mmHg}$ IOP or $\geq 20.0 \%$ IOP reduction from baseline values, although one author utilized the $810 \mathrm{~nm}$ diode laser ${ }^{12}$ and the other only targeted the inferior meshwork. ${ }^{15}$ Our findings were also less efficacious than some reports, but had a smaller sample size ${ }^{11}$ and included shorter intervals of follow-up. ${ }^{16}$

A recent meta-analysis of SLT revealed a $6.9 \%-35.9 \%$ reduction in IOP, with our results for both MLT and SLT falling within the middle of this range. ${ }^{17}$ Another recent meta-analysis of SLT vs ALT demonstrated the number of patients achieving $\geq 3 \mathrm{mmHg}$ or $\geq 20.0 \%$ decrease from baseline was $40 \%-85 \%$ of patients with a summative result of $60.1 \%{ }^{18}$ However, the baseline mean pretreatment IOP 
in these studies ranged from 19.2 to $24.0 \mathrm{mmHg}$, while our baseline IOP measurements were lower at $18.26 \mathrm{mmHg}$ in the MLT group and $16.85 \mathrm{mmHg}$ in the SLT group. ${ }^{19-22}$

One novel aspect was the post-laser survey. The MLT group experienced significantly less pain both during and after laser trabeculoplasty $(P=0.005)$. Although patients in the SLT group experienced a relatively low level of pain, the significantly decreased level of discomfort among the micropulse patients is an important distinction. Overall, more patients failed with the MLT therapy over 1 year, but this was not statistically significant and will be elucidated with future studies.

Although both of these laser therapies are efficacious, the equipment can be costly. Lee and Hutnik ${ }^{23}$ discovered a moderate cost saving if SLT was used as a primary therapy instead of topical medications under the Ontario Health Insurance Plan. Seider et $\mathrm{al}^{24}$ noted that under American insurance plans, primary SLT is less costly than most brand name medications and likely cheaper than most generic medications. When considering the cost-efficiency of SLT vs MLT, one must also evaluate their practice setting and multiuse capability of the MLT $577 \mathrm{~nm}$ yellow laser for retinal pigment epithelial stimulation, retinal photocoagulation, laser peripheral iridotomy/iridoplasty, laser trabeculoplasty, and laser suture lysis. Given the increased popularity of MLT in retinal and glaucoma practices, the cross-capability of this device has much appeal. ${ }^{25,26}$

This study includes several limitations. Prior laser treatments may confound the results in both groups, in addition to the lack of a washout period. These prior laser and medical treatments may also explain the relatively low success rate of trabeculoplasty in both groups compared to other studies. ${ }^{11,16}$ The examiners and patients were not blinded to the laser treatments, although this was offset by the strict, set randomization sequence. In addition, there was a small sample size included, yet valuable data were obtained to help guide practitioners and future studies for MLT.

\section{Conclusion}

MLT is a novel therapy in the treatment of open-angle glaucoma. Results from our head-to-head comparison between SLT and MLT provide evidence that MLT is similar to SLT in delivering substantial IOP reduction over a 52-week follow-up period, while providing less discomfort both during and after treatment.

\section{Data sharing statement}

Data for this study may be obtained from Dr David Belyea, the corresponding author at dbelyea@mfa.gwu.edu. There are no unpublished data from this protocol available in the data set.

\section{Acknowledgment}

We would like to acknowledge Dr Mansour Armaly and Family Glaucoma Research Fund for helping with the funding for this work.

\section{Disclosure}

The authors report no conflicts of interest in this work.

\section{References}

1. Wise JB, Witter SL. Argon laser therapy for open-angle glaucoma. A pilot study. Arch Ophthalmol. 1979;97(2):319-322.

2. The glaucoma laser trial (GLT) and glaucoma laser trial follow-up study: 7 results. Glaucoma Laser Trial Research Group. Am J Ophthalmol. 1995;120(6):718-731.

3. Latina MA, Park C. Selective targeting of trabecular meshwork cells: in vitro studies of pulsed and CW laser interactions. Exp Eye Res. 1995; 60(4):359-371.

4. Latina MA, Sibayan SA, Shin DH, Noecker RJ, Marcellino G. Q-switched 532-nm Nd:YAG laser trabeculoplasty (selective laser trabeculoplasty): a multicenter, pilot, clinical study. Ophthalmology. 1998;105(11):2082-2088; discussion 2089-2090.

5. Katz LJ, Steinmann WC, Kabir A, et al; SLT/Med Study Group. Selective laser trabeculoplasty versus medical therapy as initial treatment of glaucoma: a prospective, randomized trial. J Glaucoma. 2012; 21(7):460-468.

6. Pankratov MM. Pulsed delivery of laser energy in experimental thermal retinal photocoagulation. Proc Soc Photo Opt Instrum Eng. 1990;1202:205-213.

7. Kozak I, Luttrull JK. Modern retinal laser therapy. Saudi J Ophthalmol. 2015;29(2):137-146.

8. Kramer TR, Noecker RJ. Comparison of the morphologic changes after selective laser trabeculoplasty and argon laser trabeculoplasty in human eye bank eyes. Ophthalmology. 2001;108(4):773-779.

9. Fudemberg SJ, Myers JS, Katz LJ. Trabecular meshwork tissue examination with scanning electron microscopy: a comparison of micropulse diode laser (MLT), selective laser (SLT), and argon laser (ALT) trabeculoplasty in human cadaver tissue. Invest Ophthalmol Vis Sci. 2008;49:1236. Presented at the ARVO annual meeting in 2008, Fort Lauderdale, FL, USA.

10. Ingvoldstad DD, Krishna R, Willoughby L. Micropulse diode laser trabeculoplasty versus argon laser trabeculoplasty in the treatment of open-angle glaucoma. Invest Ophthalmol Vis Sci. 2005;46:123. Presented at the ARVO annual meeting in 2005, Fort Lauderdale, FL, USA.

11. Fea AM, Bosone A, Rolle T, Brogliatti B, Grignolo FM. Micropulse diode laser trabeculoplasty (MDLT): a phase II clinical study with 12 months follow-up. Clin Ophthalmol. 2008;2(2):247-252.

12. Rantala E, Välimäki J. Micropulse diode laser trabeculoplasty - 180degree treatment. Acta Ophthalmol. 2012;90(5):441-444.

13. Babalola OE. Micropulse diode laser trabeculoplasty in Nigerian patients. Clin Ophthalmol. 2015;9:1347-1351.

14. Detry-Morel M, Muschart F, Pourjavan S. Laser Mdiode, $(810 \mathrm{~nm})$ versus argon laser trabeculoplasty in the treatment of open-angle glaucoma: comparative short-term safety and efficacy profile. Bull Soc Belge Ophtalmol. 2008;(308):21-28.

15. Chudoba T, Sempińska-Szewczyk J, Filipiak E, Malukiewicz G, Shein A. Micropulse $577 \mathrm{~nm}$ laser trabeculoplasty - a prospective pilot study. Klin Oczna. 2014;116(3):174-179.

16. Lee JW, Yau GS, Yick DW, Yuen CY. MicroPulse laser trabeculoplasty for the treatment of open-angle glaucoma. Medicine. 2015; 94(49):e2075. 
17. Wong MO, Lee JW, Choy BN, Chan JC, Lai JS. Systematic review and meta-analysis on the efficacy of selective laser trabeculoplasty in open-angle glaucoma. Surv Ophthalmol. 2015;60(1):36-50.

18. Wang H, Cheng JW, Wei RL, Cai JP, Li Y, Ma XY. Meta-analysis of selective laser trabeculoplasty with argon laser trabeculoplasty in the treatment of open-angle glaucoma. Can J Ophthalmol. 2013;48(3):186-192.

19. Liu Y, Birt CM. Argon versus selective laser trabeculoplasty in younger patients: 2-year results. J Glaucoma. 2012;21(2):112-115.

20. Damji KF, Bovell AM, Hodge WG, et al. Selective laser trabeculoplasty versus argon laser trabeculoplasty: results from a 1-year randomised clinical trial. Br J Ophthalmol. 2006;90(12):1490-1494.

21. Martinez-de-La-Casa JM, Garcia-Feijoo J, Castillo A, et al. Selective vs argon laser trabeculoplasty: hypotensive efficacy, anterior chamber inflammation, and postoperative pain. Eye. 2004;18(5):498-502.
22. Almeida ED, Pinto LM, Fernandes RA, Prata TS. Pattern of intraocular pressure reduction following laser trabeculoplasty in open-angle glaucoma patients: comparison between selective and nonselective treatment. Clin Ophthalmol. 2011;5:933-936.

23. Lee R, Hutnik CM. Projected cost comparison of selective laser trabeculoplasty versus glaucoma medication in the Ontario Health Insurance Plan. Can J Ophthalmol. 2006;41(4):449-456.

24. Seider MI, Keenan JD, Han Y. Cost of selective laser trabeculoplasty vs topical medications for glaucoma. Arch Ophthalmol. 2012;130(4): 529-530.

25. Bhagat N, Zarbin M. Recent innovations in medical and surgical retina. Asia Pac J Ophthalmol (Phila). 2015;4(3):171-179.

26. Yun SH, Adelman RA. Recent developments in laser treatment of diabetic retinopathy. Middle East Afr J Ophthalmol. 2015;22(2):157-163.
Clinical Ophthalmology

\section{Publish your work in this journal}

Clinical Ophthalmology is an international, peer-reviewed journal covering all subspecialties within ophthalmology. Key topics include: Optometry; Visual science; Pharmacology and drug therapy in eye diseases; Basic Sciences; Primary and Secondary eye care; Patient Safety and Quality of Care Improvements. This journal is indexed on

\section{Dovepress}

PubMed Central and CAS, and is the official journal of The Society of Clinical Ophthalmology (SCO). The manuscript management system is completely online and includes a very quick and fair peer-review system, which is all easy to use. Visit http://www.dovepress.com/ testimonials.php to read real quotes from published authors. 